\title{
Aplicabilidade do uso de tecnologia móvel para registros em saúde durante a assistência pré-natal
}

\author{
Applicability of the use of mobile technology for health records during prenatal care
}
Aplicabilidad del uso de tecnologia móvil para registros de salud durante la atención prenatal

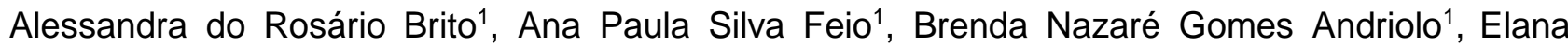
Cristina da Silva Penha ${ }^{1}$, Juliane Costa Santos ${ }^{1}$, Manuela Fernanda Medeiros de Andrade Nobre ${ }^{1}$, Matheus de Castro Santis ${ }^{1}$, Rafael Augusto Jesus Arruda ${ }^{1 *}$, Raphaela Thaís Santana Pinheiro Feio ${ }^{1}$, Samuel da Silva Ribeiro'.
\end{abstract}

\section{RESUMO}

Objetivo: Desenvolver uma ferramenta utilizando tecnologia móvel em saúde a fim de testar a aplicabilidade do mesmo durante a assistência pré-natal. Métodos: Foi realizado um estudo do tipo descritivo e transversal, com caráter quantitativo. Os participantes da pesquisa assistiram a um vídeo explicativo sobre o funcionamento do aplicativo e após disso foi aplicado um questionário adaptado a partir do Sistema Usabilyty Scala (SUS). Os dados colhidos foram analisados, através de métodos gráficos e/ou numéricos, disponíveis no software Microsoft $\AA$ Word e Excel e ajudaram a aperfeiçoar 0 aplicativo. Resultados: Participaram do estudo 14 médicos com experiência na assistência pré-natal. De acordo com os dados colhidos, $100 \%$ dos participantes afirmaram que usariam o aplicativo demonstrado e acreditam que ele seria útil na assistência pré-natal, $64 \%$ acreditam que o app é de uso fácil, e 36\% acreditam que podem necessitar de ajuda durante o uso dele. Conclusão: $O$ desenvolvimento de uma ferramenta digital para armazenamento das informações clínicas da consulta pré-natal parece ter importância para a equipe médica durante a assistência pré-natal.

Palavras-chave: Pré-natal, Aplicativos móveis, Controle de registro.

\begin{abstract}
Objective: To develop a tool using mobile health technology in order to test its applicability during prenatal care. Methods: A quantitative, descriptive and cross-sectional study was carried out. The research participants watched an explanatory video about the application's operation and after that, a questionnaire adapted from the Usabilyty Scala System (SUS) was applied. The collected data were analyzed, using graphic and / or numerical methods, available in Microsoft $\circledast$ Word and Excel software and helped to perfect the application. Results: 14 physicians with experience in prenatal care participated in the study. According to the data collected, $100 \%$ of the participants stated that they would use the demonstrated application and believe that it would be useful in prenatal care, $64 \%$ believe that the app is easy to use, and $36 \%$ believe that they may need help during the use of it. Conclusion: The development of a digital tool for storing the clinical information of the prenatal consultation seems to have importance for the medical team during the prenatal care.
\end{abstract}

Key words: Prenatal care, Mobile apps, Record control.

${ }^{1}$ Centro Universitário Metropolitano da Amazônia (UNIFAMAZ), Belém - PA.

*E-mail: arrudamed92@gmail.com

SUBMETIDO EM: 2/2021

ACEITO EM: 3/2021

PUBLICADO EM: 3/2021 


\section{RESUMEN}

Objetivo: Desarrollar una herramienta que utilice tecnología de salud móvil para probar su aplicabilidad durante la atención prenatal. Métodos: se realizó un estudio cuantitativo, descriptivo y transversal. Los participantes de la investigación vieron un video explicativo sobre el funcionamiento de la aplicación y posteriormente se aplicó un cuestionario adaptado del Usabilyty Scala System (SUS). Los datos recolectados fueron analizados mediante métodos gráficos y / o numéricos, disponibles en software Microsoft@ Word y Excel y ayudó a perfeccionar la aplicación. Resultados: participaron del estudio 14 médicos con experiencia en atención prenatal. Según los datos recopilados, el 100\% de las participantes afirmaron que usarían la aplicación demostrada y creen que sería útil en la atención prenatal, el $64 \%$ cree que la aplicación es fácil de usar y el $36 \%$ cree que puede necesitar ayuda. durante el uso de la misma. Conclusión: El desarrollo de una herramienta digital para almacenar la información clínica de la consulta prenatal parece tener importancia para el equipo médico durante la atención prenatal.

Palabras clave: Prenatal, Aplicaciones móviles, Control de registro.

\section{INTRODUÇÃO}

Regularizado pela Portaria $n^{\circ} 570$ de $1^{\circ}$ de junho de 2000, o Programa de Humanização no Pré-natal e Nascimento, diante da necessidade de estabelecer mecanismos de melhoria ao acesso, ampliação da cobertura e da qualidade do acompanhamento pré-natal e a realização do cadastramento das gestantes, foi implementado pelo SUS, sendo este responsável por assegurar tais direitos (BRASIL, 2000).

Estudos que avaliam a qualidade no atendimento pré-natal revelam que uma atenção pré-natal de qualidade é capaz de diminuir entraves sociais, como a morbidade e a mortalidade materno-infantil, seguindo as evidências científicas que a correta e minuciosa identificação do risco gestacional pelos profissionais de atendimento pré-natal permite a orientação e os encaminhamentos adequados em cada fase da gestação, assegurando uma gravidez tranquila (LENNON S, et al., 2015; TOMASI E, et al., 2017; WOLDE F, et al., 2018). Outrossim, a assistência imprópria do pré-natal é uma realidade nacional e há inúmeros fatores que podem justificar, como localização geográfica, com áreas de difícil acesso e baixa escolaridade.

Nesse viés, um estudo realizado no Canadá relatando a inadequada adesão ao pré-natal, cita que mesmo havendo o sistema universal de saúde e não sendo necessário pagar pelas consultas, há inúmeras barreias para que tal evento ocorra como: barreiras econômicas, psicossociais e estruturais que influenciam diretamente ao cuidado dessas mulheres e adição aos problemas familiares, estresse, gravidez não planejada, custos com transporte e cuidados infantis, além de não saber onde obter informações ou cuidados ou longa espera pelo atendimento, e medo de apreensão da criança pela agência de bem-estar da criança (MAUREE I, et al., 2019).

Sobre o assunto, múltiplos estudos citam que a inadequada adesão ao pré-natal está associada a indicadores individuais de desvantagem social, como baixa renda, educação para o ensino fundamental como máximo grau de escolaridade, ser mãe solteira e ser viver em área isolada. Logo, a associação entre assistência imprópria ao pré-natal e desvantagem social é semelhante aos achados de outros países desenvolvidos como Bélgica, Nova Zelândia e Inglaterra, não sendo somente uma realidade de países em desenvolvimento (ROSAS LG, et al., 2014).

Assim, com a utilização de tecnologias móveis, capazes de armazenar informações de saúde da gestante, poderão auxiliar o médico e/ou profissional de saúde para melhor acompanhamento, planejamento terapêuticos e/ou condutas e atingir melhores desfechos na gestação. A acessibilidade precária somada à má adesão ao pré-natal aumenta os fatores de risco tanto para a mãe quanto para a criança. Consequentemente, as informações do pré-natal são perdidas e/ou mal coletadas. Certamente 0 profissional de saúde necessita do registro de informações da gestante de modo mais acessível, claro e conciso, para que promova o acompanhamento adequado da gestação (LUND S, et al., 2016). 
Um estudo realizado em Gana, o qual testou a aplicabilidade de um dispositivo de apoio à decisão diagnóstica, fez sugestão à conduta. Porém, o julgamento clínico final é exclusivo do profissional da área da saúde (KWAK MY, et al., 2018). Logo, os dispositivos vêm com intuito de auxiliar os profissionais, para que as iatrogênicas e omissões sejam evitadas. Um estudo piloto na Califórnia, EUA, onde se implementou totens eletrônicos em salas de espera de consultas pré-natais, demonstrou ser uma estratégia viável para fornecer educação em saúde ambiental e de baixo custo para mulheres grávidas, latinas e de pouca escolaridade e renda.

O referido estudo conseguiu fornecer educação com informativos e jogos do tipo Quiz para avaliação do conhecimento das gestantes e propor soluções e dicas de assistência pré-natal. Consequentemente, o desfecho mostrou que o nível de aceitação por parte das gestantes foi alto, e que por ser um meio eletrônico, ainda que essas gestantes tivessem pouca escolaridade, foi eficaz como ferramenta educacional tanto para as gestantes como, também, ferramenta de coleta ao centro de serviço gerando um banco de dados a ser utilizado como estratégia em saúde (KWAK MY, et al., 2018).

Em Zanzibar, na Tanzânia, a utilização de tecnologias móveis por grávidas durante o pré-natal aumentou significativamente a proporção de mulheres que recebem as visitas obrigatórias do pré-natal, as mínimas recomendadas durante a gravidez (CHHEA C, et al., 2018). Além de haver uma tendência na melhoria do cuidado na gestação. A princípio, o mesmo estudo provou que tecnologias de aplicativos móveis podem contribuir para a melhoria da saúde materna e neonatal e devem ser consideradas pelos agentes de políticas públicas no trato com esse momento materno.

À vista disso, percebe-se que o advento das Tecnologias de Informação e Comunicação (TIC), foi discretamente implementada no Brasil e sua obtenção é dificultada pelo excesso de entraves burocráticos. No entanto, fica evidenciado que ferramentas em TIC, se utilizadas de maneira integrada e acessível, podem estimular e disseminar educação em saúde, no usufruto de ferramentas simultâneas de áudio e vídeo que possibilitem o processo integrativo das tecnologias atuais em registro pré-natal (SILVA TIM, et al., 2018).

Portanto, ainda que o Ministério da Saúde tenha lançado em 2014 as Diretrizes Nacionais de Implantação da Estratégia e-SUS na Atenção Básica numa tentativa de mesclar essas diretrizes com a Política Nacional de Informação e Informática em Saúde (PNIIS), no sentido de integrar os sistemas de informações em saúde do SUS, pouco se avançou quando o assunto são tecnologias móveis e que facilitem, por exemplo, o acompanhamento pré-natal (MOTA DNM, et al., 2018). A partir de estudos apontados em outros países, das mais distintas estratificações sociais e econômicas, a implementação das informatizações em saúde é de relevante utilidade ao garantir a segurança e o fácil acesso das informações colhidas durante toda a gestação (GUO Y, et al., 2018).

O objetivo do estudo foi desenvolver uma ferramenta utilizando tecnologia móvel em saúde a fim de testar a aplicabilidade do mesmo durante a assistência pré-natal.

\section{MÉTODOS}

O estudo foi realizado no Centro Universitário Metropolitano da Amazônia, na cidade de Belém-PA, por meio de solicitação da lista de profissionais da saúde que trabalham na assistência pré-natal, no período de 2020. Foi realizado um estudo do tipo descritivo e transversal, com caráter quantitativo.

Para o recrutamento da amostra, foi solicitada uma lista a coordenação do curso de medicina, contendo o nome de todos os docentes envolvidos na assistência pré-natal, independente do módulo ministrado pelo profissional e que tenham lotação no semestre 2020 no curso em questão do Centro Universitário Metropolitano da Amazônia. A amostra foi composta por população de 14 docentes, que atuam no ensino e na assistência pré-natal.

Foram incluídos todos os docentes que atuam ou atuaram na assistência pré-natal, independentes de especialidades, tempo de formação e módulo de atuação durante o exercício da atividade acadêmica. Os participantes incluídos nesta pesquisa foram estudados segundo os preceitos da Declaração de Helsinque e 
do Código de Nuremberg, seguindo as, determinações da Resolução 466/2012 do Conselho Nacional de Saúde, sendo avaliado e aprovado pelo Comitê da Ética em Pesquisa da Centro Universitário Metropolitano da Amazônia (UNIFAMAZ), cumprindo-se as orientações descritas no Regimento do Comitê Científico do Curso de Medicina, e após o aceite do orientador, da instituição.

A coleta de dados foi realizada por meio da aplicação de um questionário adaptado a partir da Sistem Usabilyty Scala (SUS), escala validada, de fácil entendimento, com questões objetivas, que avalia da usabilidade de um aplicativo para smartphone. Devido ao cenário de afastamento social, os questionários foram enviados aos participantes via Microsoft Forms, o qual tinha um vídeo que simulava o funcionamento do aplicativo, a após sua apresentação, o participante tinha acesso ao questionário acerca da usabilidade do mesmo. Foram analisados o tempo médio de formação dos médicos docentes, titulação de especialização médica, tempo de assistência ao pré-natal, idade e gênero. Quanto ao aplicativo, foi avaliado quanto ao nível usabilidade e a qualidade da interface. E foram analisadas as sugestões de melhorias.

Foram utilizados o programa Excel 2010, para a organização de dados e tabelas, e o Word 2010, para a redação do texto, e de acordo com a natureza das variáveis, foram aplicadas análises estatísticas descritivas e comparativas sendo informados os valores dos dados analisados, através de métodos gráficos e/ou numéricos, disponíveis no software Microsoft® Word e Excel, respectivamente. A pesquisa foi aprovada pelo Comitê de Ética em Pesquisa (CEP) sob número de parecer 4.178.952.

\section{RESULTADOS}

Dos 25 docentes que receberam o questionário, um total de 14 (56\%) aceitaram participar da pesquisa. (Gráfico 1). O número inferior ao tamanho amostral anteriormente calculado ocorreu por conta da pandemia da COVID-19, pois, devido o distanciamento social a coleta de dados foi feita de maneira remota.

Gráfico 1 - Nível de aceitação na participação da pesquisa "A aplicabilidade do uso de tecnologia móvel durante a assistência pré-natal".

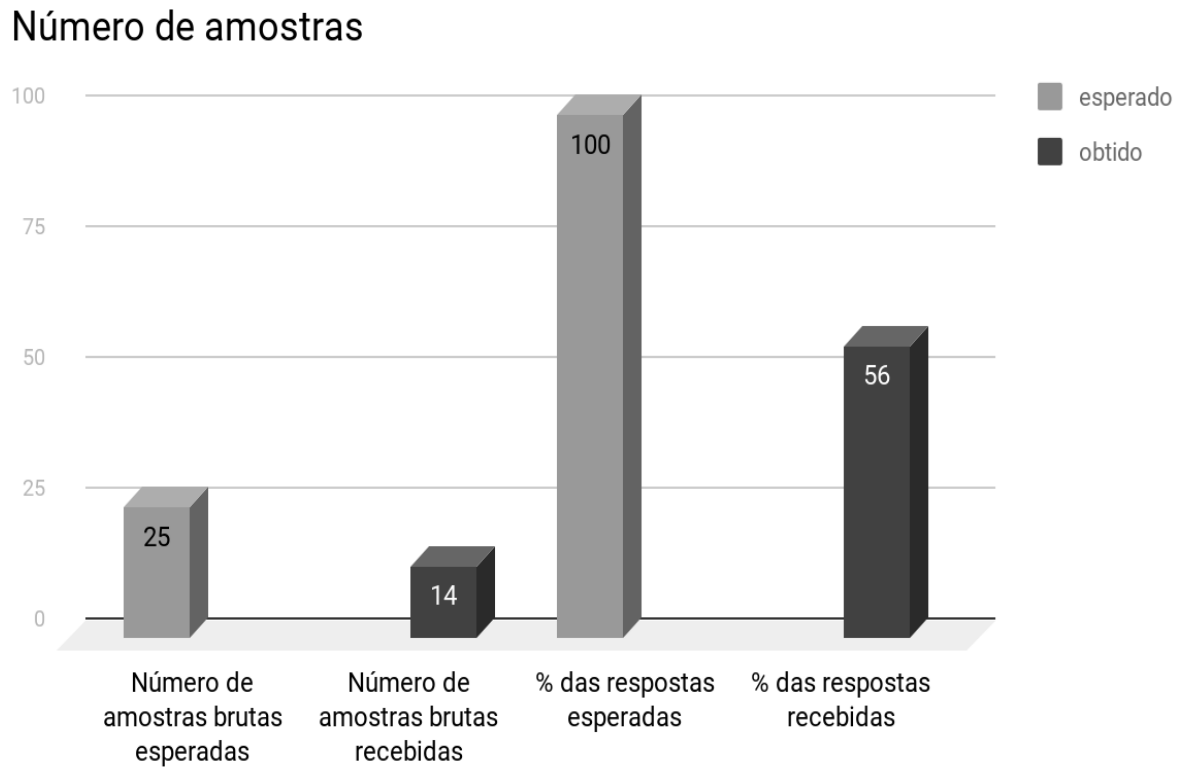

Fonte: Arruda RAJ, et al., 2021.

Em relação ao tempo de conclusão da graduação, observa-se que 50\% está formado a menos de 5 anos, $42 \%$ entre 5 e 15 anos e 7, 17\% a mais de 15 anos. Além disso, observou-se também que 57, 14\% dos entrevistados eram especializados em medicina da família e comunidade, $14,28 \%$ em ginecologia e obstetrícia, $7.17 \%$ em clínica médica e $21.4 \%$ não tinham especialização (Tabela 1). 
Quando perguntado aos participantes qual o seu gênero, dos 14 docentes, 3 responderam masculino $(21,42 \%)$ e 11 responderam feminino (78,57\%). Observou-se que o perfil da amostra converge com a maior presença feminina atuando na assistência pré-natal.

Tabela 1- Tempo de conclusão da Graduação e especialidades dos docentes envolvidos na assistência pré-natal que fazem parte do corpo docente da UNIFAMAZ em Belém/PA.

\begin{tabular}{ccc}
\hline Resposta dos Docentes & Quantidade & $\%(\mathbf{N}=\mathbf{1 4})$ \\
\hline Conclusão da Graduação(anos) & & \\
\hline$<5$ & 7 & $50,0 \%$ \\
entre 5-15 anos & 6 & $42,85 \%$ \\
$>15$ & 1 & $7.17 \%$ \\
\hline Especialidade & & $57,14 \%$ \\
\hline Medicina de Família e Comunidade & 8 & $14,28 \%$ \\
Ginecologia e obstetrícia & 2 & $7.17,0 \%$ \\
Clínica Médica & 1 & $21,24 \%$ \\
\hline Sem especialização & 3 &
\end{tabular}

Fonte: Arruda RAJ, et al., 2021.

A média da faixa etária dos participantes foi de 32 anos quando perguntado qual sua idade. Sendo as idades compreendidas entre 25-54 anos (Gráfico 2). No gráfico acima podemos observar que $100 \%$ dos participantes acreditam que o aplicativo apresentado a eles era útil durante o acompanhamento do cuidado pré-natal (Gráfico 3).

Gráfico 2 - Idade em anos, no trabalho de pesquisa "A aplicabilidade do uso de tecnologia móvel durante a assistência pré-natal".

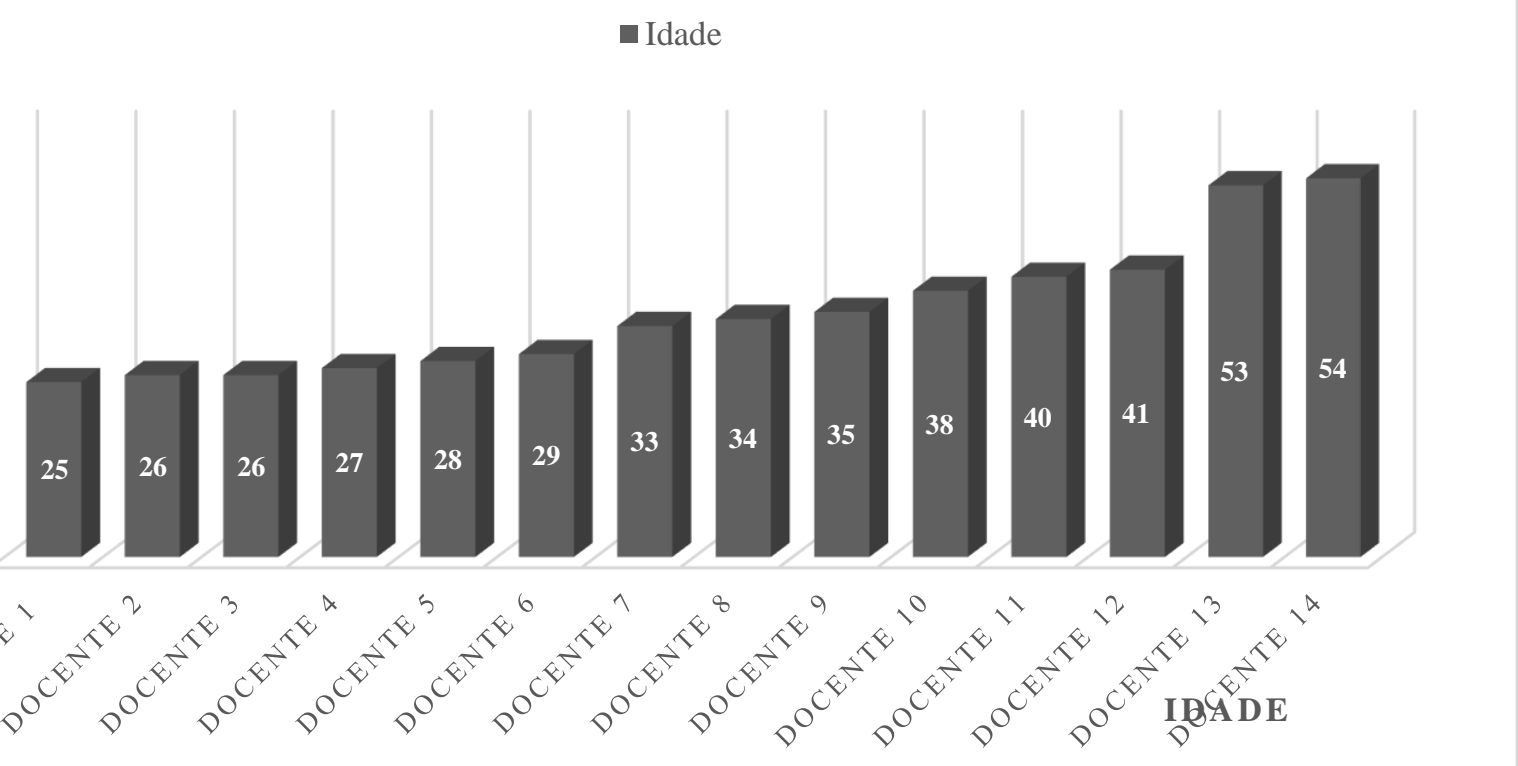

Fonte: Arruda RAJ, et al., 2021. 
Gráfico 3 - Considera o aplicativo útil para o acompanhamento das gestantes durante o cuidado pré-natal na pesquisa "A aplicabilidade do uso de tecnologia móvel durante a assistência pré-natal".

Utilidade do aplicativo para acompanhamento pré-natal

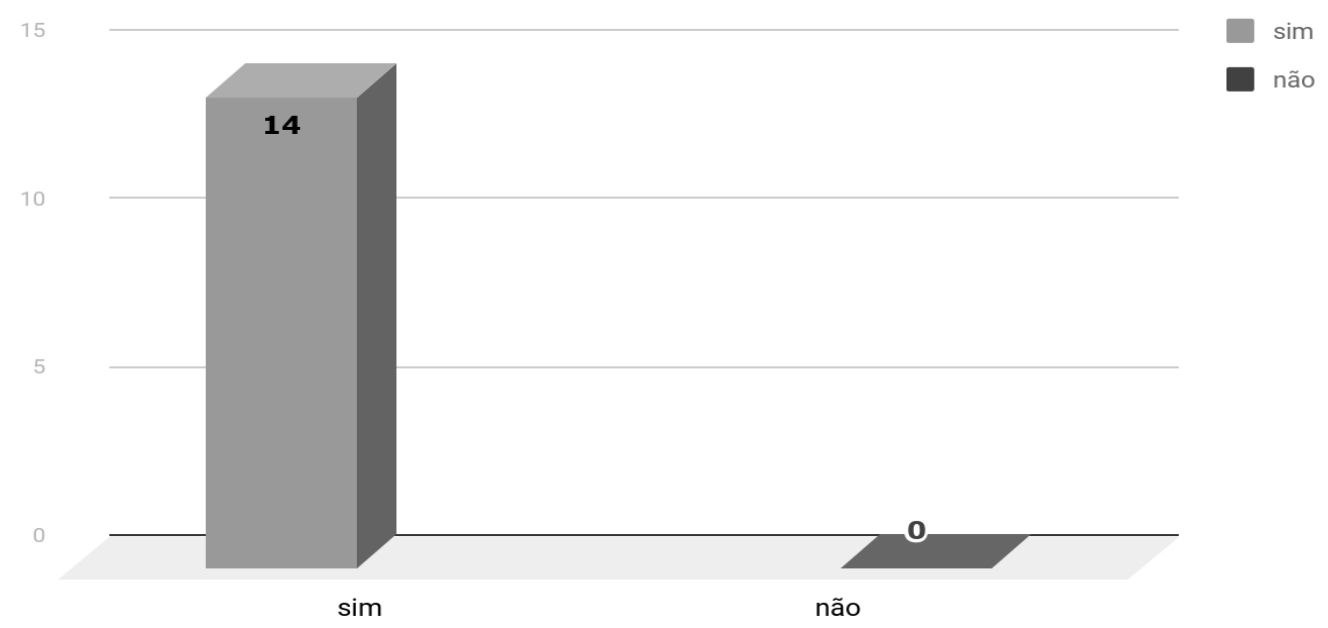

Fonte: Arruda RAJ, et al., 2021.

O Gráfico 3 demostra que 100\% dos docentes tem interesse em utilizar o aplicativo na prática da assistência pré-natal. Pois quando perguntados se gostariam de usar o aplicativo demostrado, todos os 14 participantes responderam sim. Ao serem questionados se eles consideram, a partir do vídeo exposto, se o aplicativo tem potencial para ser considerado fácil durante o uso no pré-natal. As opções de resposta dadas foram: concordo plenamente (64,3\%), concordo parcialmente (37,7\%), não concordo e nem discordo ( $0 \%)$, discordo parcialmente $(0 \%)$, e discordo plenamente $(0 \%)$.

Em relação a resposta do docente quando perguntado se acha que pode precisar de ajuda durante o uso do aplicativo na pesquisa. As opções dadas foram: Sim, 1 resposta $(7,1 \%)$. Não, 10 respostas $(71,4 \%)$.

Talvez, 3 respostas $(21,4 \%)$. Quando perguntado se acha que as várias funções do sistema estão muito bem integradas na pesquisa. As opções dadas para resposta foram: Concordo plenamente $(57,1 \%)$, concordo parcialmente (42,9\%), não concordo e nem discordo, discordo parcialmente e discordo plenamente (Gráfico 4).

Gráfico 4 - Resposta do docente quando perguntado se acha que as várias funções do sistema estão muito bem integradas na pesquisa "A aplicabilidade do uso de tecnologia móvel durante a assistência pré-natal".

as várias funções do sistema estão muito bem integradas

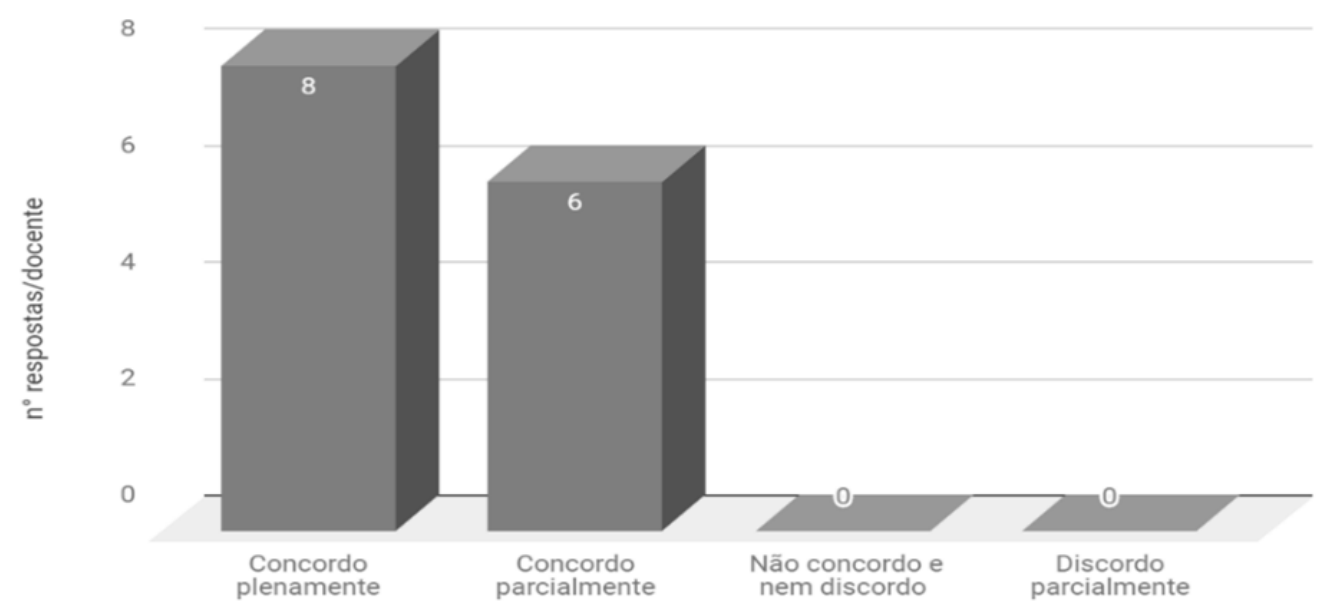

Fonte: Arruda RAJ, et al., 2021. 
Cabe citar que, quando perguntado se o docente imagina se as pessoas e/ou os docentes aprenderão como usar esse sistema rapidamente. Foram dadas as seguintes opções de resposta: concordo plenamente $(42,9 \%)$, concordo parcialmente $(50 \%)$, não sabe informar $7,1 \%)$, discordo parcialmente $(0 \%)$, e discordo plenamente (0\%) (Gráfico 5).

Gráfico 5 - Resposta do docente quando perguntado se imagina que as pessoas e/ou os docentes aprenderão como usar esse sistema rapidamente na pesquisa "A aplicabilidade do uso de tecnologia móvel durante a assistência pré-natal".

\section{As pessoas aprenderão como usar esse sistema rapidamente}

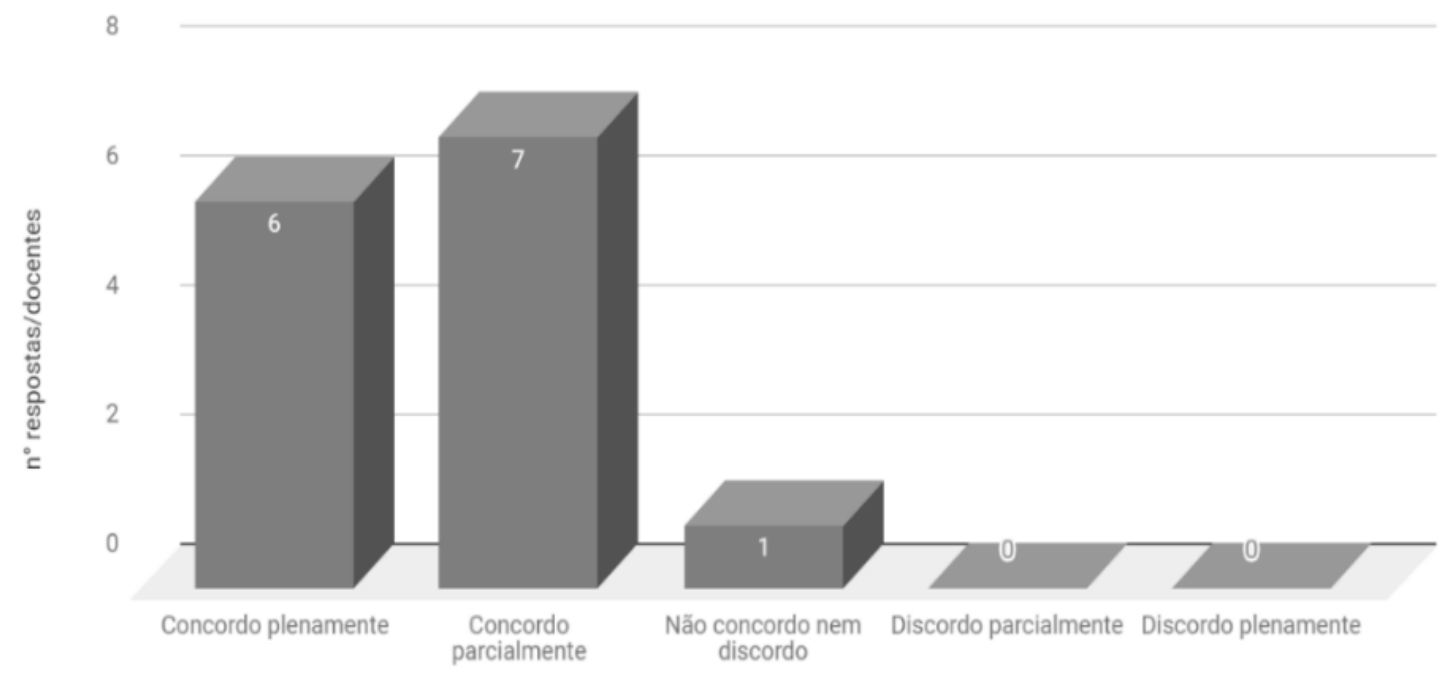

Fonte: Arruda RAJ, et al., 2021.

Com relação a indicação do aplicativo, quando questionado se indicaria o aplicativo aos serviços assistências durante o pré-natal. Tendo, 14 respostas $\operatorname{sim}(100 \%)$ e 0 respostas não $(0 \%)$.

\section{DISCUSSÃO}

O perfil da amostra converge com a maior presença feminina atuando na assistência pré-natal (78\%), confirmado pelo Ministério da Saúde que desde 2009, mostrou uma predominância da mulher nos cursos da área da saúde, com ênfase no curso de medicina (BARBOSA SP, et al., 2020).

A média da faixa etária dos participantes foi de 32 anos. Esse achado foi também descrito em outro estudo, em que a idade média de médicos atuando na saúde pré-natal está em torno de 24 e 34 anos. Pois, uma vez que formados, os profissionais buscam adquirir maior experiência trabalhando nas estratégias de saúde da família (TORRES RAM, et al., 2012).

Quando questionados quanto o tempo de conclusão de sua graduação, $50 \%$ dos entrevistados responderam, menos de 5 anos, logo, observou-se que o tempo de formação entre os docentes participantes confirma a tendência de que, assim que formado, o profissional busca atuar em seus primeiros anos na medicina de família e comunidade, adquirindo experiência para então adentrar nos cursos de especialização. Assim, tem-se um maior número de profissionais atuantes na medicina de família e comunidade (57.14\%) afinal, por estarem mais alcançáveis à população, abordam cotidianamente e de maneira inevitável a assistência pré-natal, sendo assim maior a adesão da comunidade e viabilização de inserção de tecnologias móveis nesta área (ALMEDA MM, et al., 2014).

O perfil da amostra converge com a maior presença feminina (785) atuando na assistência pré-natal, confirmado pelo Ministério da Saúde que desde 2009, mostrou uma predominância da mulher nos cursos da área da saúde, com ênfase no curso. A não adesão ao pré-natal, exemplificadas nos estudos abordados por 
este trabalho, mostram que há uma linha tênue entre a qualidade do acompanhamento e o sucesso, ou não, na efetividade do parto, uma vez que lacunas nos acompanhamentos do pré-natal dificultam seu sucesso (TORRES RAM, et al., 2012). Assim como o nível de escolaridade, o nível de acesso as tecnologias de informação em saúde, são, também marcadores sociais de efetividade no pré-natal.

Além disso, há uma importante questão em se estreitar a relação médico paciente com o advento das tecnologias integrativas em saúde, uma vez que as demasiadas informações contidas nos cartões da gestante podem ter falhas em seu registro, compreensão por parte da gestante e até mesmo omissão do corpo técnico de acompanhamento pré-natal no devido preenchimento do cartão da gestante. Os profissionais entrevistados afirmaram em sua totalidade (100\%) que acreditam na utilidade do uso do aplicativo durante a assistência pré-natal, o que corrobora com o estudo realizado em Crato-CE, onde foi analisado como os profissionais de saúde estão utilizando tecnologias digitais da comunicação e informação, pois, essas tecnologias apresentaram extrema relevância no cotidiano do trabalho desses profissionais (MEYER ED, et al., 2018).

Uma vez que, tais ferramentas auxiliam nos principais problemas de saúde enfrentados pela população, como referências e deslocamentos desnecessários dos pacientes e consequentemente, com redução dos custos tanto para o sistema de saúde, como para os usuários. Além disso, os dados de saúde do paciente disponíveis para um profissional de saúde em um smartphone, melhoram a rastreabilidade do paciente e o acompanhamento em casa, tornando possível o encaminhamento oportuno. Assim, a utilidade de tecnologias de informação e comunicação em estratégias saúde da família, é uma possibilidade de integração com equipes, maior velocidade no fluxo de informações entre as unidades básicas, quantificando e qualificando o trabalho interdisciplinar (MEYER ED, et al., 2018).

É importante relatar quando perguntados se as várias funções do sistema estão muito bem integradas na pesquisa, $57 \%$ dos entrevistados concordaram plenamente e $42,9 \%$ concordaram parcialmente. Em um estudo realizado sobre o e-SUS, percebe-se que o advento das Tecnologias de Informação e Comunicação (TIC), discretamente implementadas no Brasil, podem ser definidas como um conjunto de ferramentas que podem ser utilizadas de maneira integrada, no intuito de estimular e disseminar educação em saúde, no usufruto de ferramentas simultâneas de áudio e vídeo que possibilitem o processo integrativo das tecnologias atuais. Assim, vale salientar, que a coleta de informações precisa integralidade para facilitar no diagnóstico e tomadas de decisões.

Esses dados justificam o interesse maciço dos docentes em usar o aplicativo e em indica-lo aos serviços assistências pré-natal, pois, como observado em outro estudo, os profissionais compreendem que o uso de tecnologias no desenvolvimento do serviço possibilita o desenvolvimento de três categorias: Organização e melhoria no processo de trabalho; Acesso ao conhecimento e atualização e Planejamento de atividades na Estratégia saúde da família. Além disso, os médicos são os profissionais que mais utilizam a internet no ambiente de trabalho e o telefone móvel é a tecnologia mais utilizada por profissionais da área da saúde, sendo médicos e enfermeiros os que mais utilizam essa ferramenta.

Afinal, a adoção de tecnologias como telefone móvel, tecnologia mais antiga simples e barata, podendo ser utilizados em pontos remotos, trazendo aplicabilidade e apoio. Ademais, 64,3\% dos médicos entrevistados na pesquisa, consideram, a partir do vídeo exposto, que 0 aplicativo tem potencial para ser considerado fácil durante o uso no pré-natal, $43 \%$ concordam plenamente que as pessoas aprenderão como usar esse sistema rapidamente e $71,4 \%$ acreditam que não precisariam de ajuda para utilizar o aplicativo. O que é extremamente importante acompanhamento da gestação, já que, o acesso fácil manuseio e acesso as informações colhidas durante o pré-natal, além de auxiliam os médicos nas tomadas de decisão frente a uma complicação (ALMEDA MM, et al., 2014).

Além disso, o acesso descomplicado a esses dados pode ser utilizado pelas gestantes como ferramenta de autocuidado, pois, mulheres mais bem informadas tentem a cuidar melhor da sua saúde. Assim, as transformações exigem que os profissionais se apropriem das tecnologias, utilizando-as como meio de superar problemas de forma rápida e eficiente, mas para que seu uso seja adequado, faz-se necessário 
desenvolver competências e habilidades. O uso de tecnologias em saúde vem sendo disseminado, pelas universidades, fortalecendo a sua utilização também como ferramentas de formação profissional (ALMEDA MM, et al., 2014).

Dessa maneira, e para a segurança das informações da gestante, faz-se necessário a informatização das informações em saúde trocadas durante a assistência pré-natal, garantindo uma maior cobertura dos eventos pontuados pelo Ministério da Saúde como calendário vacinal, medidas de crescimento uterino e outros importantes marcadores da gestação (SILVEIRA RP, et al., 2014). Pelo mesmo prisma, a pesquisa "a aplicabilidade do uso de tecnologia móvel para registros em saúde durante a assistência pré-natal" conseguiu demonstrar que quando perguntado ao docente sobre a integração das diversas funções do aplicativo elaborado, a maioria dos pesquisados afirmaram que tal organização corroboravam para seu fácil uso e aplicabilidade durante a assistência pré-natal.

Nestes fins, os resultados observados pela pesquisa supracitada estão em consonância com o investigado em outras pesquisas no mesmo sentido, como um estudo feito para demonstrar a integração dos cuidados primários e orientações aos agentes de saúde na África do sul, onde a informatização e uso de tecnologias móveis resultou em melhor compreensão e aceitação por parte das gestantes a respeitos do conhecimento acerca do pré-natal e, também, demonstrando que os dados de saúde do paciente disponíveis para um profissional de saúde em um smartphone como parte do acompanhamento pré-natal melhoram a rastreabilidade do paciente e 0 acompanhamento em casa, tornando possível 0 encaminhamento oportuno (MACHADO CDB, et al., 2018).

Ao questionar o docente quanto as suas sugestões para a melhoria do aplicativo, obteve-se em sua maioria respostas positivas em relação a interface do aplicativo com qualidade nas funções o tornando de fácil manuseio. Tal correlação pode ser feita de maneira oportuna com pesquisas que asseguram que, quanto melhor e mais interativa a interface do aplicativo, melhor sua adesão e acessibilidade $7,11,12,14$. Em contrapartida, foi sugerido por alguns pesquisados melhorias no "web designer" do aplicativo e ter a opção "digitalizar imagens laboratoriais e ultrassonográficas.

Quanto ao "web designer, por ser uma iniciativa tomada por discentes que não tem formação ou conhecimento técnico profundo na área, e ser uma pesquisa de custeada pelos pesquisadores, o serviço de desenvolvimento do layout foi construído pelos próprios pesquisadores, com a melhor viabilidade possível já demonstrada com pesquisa. Assim, a contratação futura ou mesmo parcerias serão avaliadas para melhorias do aplicativo, o que mesmo assim não configura, dadas as respostas positivas do assunto em si, um agente limitante para a evolução da ferramenta por se tratar de uma tecnologia móvel e esta sempre carecer de aprimoramentos, como também inserir a opção de digitalizar imagens.

Portanto, reforça-se que, pesquisas em tecnologias móveis são ainda escassas e, apesar do avanço na usabilidade e aplicabilidade de aplicativos móveis em assistência pré-natal existe uma necessidade de pesquisas mais robustas para nortear os pesquisadores com interesse na área. As pesquisas levantadas em sua maioria deixam evidente a carência de investimento de setores governamentais e privados em trabalhos desse tipo (TEÓFILO TJ, et al., 2017). É essencial investir na montagem, teste e capacitação profissional, para que se possam operacionalizar os aplicativos móveis na coleta de consultas de acompanhamento pré-natal.

\section{CONCLUSÃO}

O modelo adaptado do aplicativo para assistência pré-natal, apresentado em forma de vídeo explicativo, consiste em um software de coleta automatizada. Tornando o acesso aos dados da consulta mais rápido e fácil. Esse aplicativo eliminaria as controvérsias de uma ficha mal preenchida. Afinal, todas as informações serão integradas e podem ser armazenada com segurança em uma nuvem, o que possibilita um controle em tempo real. Logo, pode-se inferir que a partir do plano de construção proposto, o desenvolvimento de uma ferramenta digital para armazenamento das informações clínicas da consulta durante o período de gestação é de grande valia para assistência pré-natal. Apesar disso, são necessárias mais pesquisas para melhor construção de uma elaboração deste aplicativo de dispositivo móvel. 


\section{REFERÊNCIAS}

1. ABEJINRINDE IO, et al. Viability of diagnostic decision support for antenatal care in rural settings: findings from the Bliss4Midwives Intervention in Northern Ghana. J Glob Health, 2019; 9(1): 010420.

2. ALMEDA MM, et al. O uso de tecnologias da informação e comunicação em áreas rurais é suficiente para a educação continuada? J Bras TeleSSaúde, 2014; 3(1): 211-9.

3. BARBOSA SP, et al. Aspectos que Compõem o Perfil dos Profissionais Médicos da Estratégia Saúde da Família: o Caso de um Município Polo de Minas Gerais. Revista Brasileira de Educação Médica, 2020; 43(1, Suppl. 1): 395403.

4. BRASIL. Ministério da Saúde. Gabinete do Ministro. Portaria ํo 570, de 1ำ de Junho de 2000 [documento na internet]. Brasília (DF): Ministério da Saúde; 2000.

5. CHHEA C, et al. Low birth weight of institutional births in Cambodia: Analysis of the Demographic and Health Surveys 2010-2014. PLoS One, 2018; 13(11): e0207021.

6. GUO Y, et al Pregnant and Postpartum Women's Access and Use of Their Health Records. MCN Am J Matern Child Nurs., 2018; 43(3): 164-170.

7. KWAK MY, et al. Accessibility of Prenatal Care Can Affect Inequitable Health Outcomes of Pregnant Women Living in Obstetric Care Underserved Areas: a Nationwide Population-Based Study. JKorean Med Sci., 2018; 34(1): e8.

8. LENNON S, et al. The effectiveness of interventions to improve access to and utilization of prenatal care: a systematic review protocol. JBI Database of Systematic Reviews \& Implementation Reports, 2015; 13(5): 10-23.

9. LUND S, et al. Mobile phones improve antenatal care attendance in Zanzibar: a cluster randomized controlled trial. BMC Pregnancy Childbirth, 2016; 17(14): 29.

10. MACHADO CDB. Educação Médica no Brasil: uma Análise Histórica sobre a Formação Acadêmica e Pedagógica. Rev. bras. educ. med., Brasília, 2018; 42(4): 66-73.

11. MAUREEN I, et al. The Association of Inadequate and Intensive Prenatal Care With Maternal, Fetal, and Infant Outcomes: A Population-Based Study in Manitoba, Canada. Journal: Journal of Obstetrics and Gynaecology Canada, 2019.

12. MEYER ED, et al. Why high tech needs high touch: Supporting continuity of community primary health care. Afr J Prim Health Care Fam Med., 2018; 10(1): e1-e6.

13. MOTA DN, et al. Tecnologias da informação e comunicação: influências no trabalho da estratégia Saúde da Família Information and communication technologies: influences in the work of the Family Health strategy. 2018; 17(2): 2030.

14. ROSAS LG, et al. Acceptability of health information technology aimed at environmental health education in a prenatal clinic. Patient Educ Couns. 2014; 97(2): 244-7.

15. SILVEIRA RP. Crise na educação médica? Um ensaio sobre o referencial arendtiano. Interface - Comunicação, Saúde, Educação [online], 2014; 18(48): 115-126.

16. SILVA TIM, et al. Difusão da inovação e-SUS Atenção Básica em Equipes de Saúde da Família. Rev. Bras. Enferm. [Internet], 2018; 71(6): 2945-2952.

17. TEÓFILO TJS. Apostas de mudança na educação médica: trajetórias de uma escola de medicina. Interface Comunicação, Saúde, Educação [online], 2017; 21(60): 177-188.

18. TOMASI E, et al. Qualidade da atenção pré-natal na rede básica de saúde do Brasil: indicadores e desigualdades sociais. Cad. Saúde Pública [Internet], 2017; 33(3): e00195815.

19. TORRES RAM, et al. Tecnologias digitais e educação em enfermagem: a utilização da web-rádio como estratégia pedagógica. J.Health Inform., 2012; 4(Especial): 1-5.

20. WOLDE F, et al. Determinants of late initiation for antenatal care follow up: the case of northern Ethiopian pregnant women. BMC Res Notes, 2018; 11(1): 837. 